\title{
Erratum
}

\section{Errata for Formal Aspects of Computing (2006) 18:495-517 and their consequences}

\author{
Matthew Collinson, David Pym and Chris Tofts \\ Hewlett-Packard Laboratories, Filton Road, Stoke Gifford, Bristol BS34 8QZ, UK. \\ E-mail: matthew.collinson@hp.com; david.pym@hp.com; chris.tofts@hp.com
}

We present a correction for an error that occurs in the following paper Formal Aspects of Computing (2006) 18:495-517.

At first sight, the error appears to be simply a misplaced quantifier in the definition of bisimulation. We explain, however, that the error and its correction reveal a subtle interaction between the substructural connectives of MBI and the resource-process calculus SCRP.

We begin with a specific example which illustrates the error. We include also the known typographical errors. We include also a statement of the consequences of these errata for the paper Electronic Notes in Theoretical Computer Science 172, 545-587, 2007, which builds directly upon Formal Aspects of Computing (2006) 18:495-517 and which illustrates the significance of these errata.

\section{Example}

We begin with an example that illustrates an error in the definition of bisimulation used in [PT06].

We start with the resource monoid $(\mathbb{N},+, 0,=)$ and the action monoid

$$
A c t=\left\{i^{p} z^{q} \mid p, q \in \mathbb{N}\right\}
$$

generated freely from two primitive actions $z$ and $i$. Note that, for simplicity, we take here just individual resources rather than sets of resources. This is an inessential change with no impact on the theory.

We take

$$
\mu\left(i^{p} z^{q}, n\right)= \begin{cases}n+p & \text { if } p \neq 0 \\ 0 & \text { if } n=p=0 \\ \uparrow & \text { if } n \neq p=0\end{cases}
$$

for all $n \in \mathbb{N}$. In particular, write $1=i^{0} z^{0}$. This defines a modification function. Note that $i$ is the incrementation action whilst $z$ says "if zero then tick".

Consider the processes $E$ and $F$ defined by

$$
\begin{array}{ll}
E=i: E^{\prime} & E^{\prime}=i: E+z: F \\
F=i: F^{\prime} & F^{\prime}=F
\end{array}
$$

The online version of the original article can be found under doi:10.1007/s00165-006-0018-z. 
using auxilliary processes $E^{\prime}$ and $F^{\prime}$. These processes generate transition structures that look as follows:

$$
\begin{aligned}
& n, E \stackrel{i}{\rightarrow} n+1, E^{\prime} \stackrel{i}{\rightarrow} n+2, E \stackrel{i}{\rightarrow} \cdots \\
& n, F \stackrel{i}{\rightarrow} n+1, F^{\prime} \stackrel{i}{\rightarrow} n+2, F \stackrel{i}{\rightarrow} \cdots
\end{aligned}
$$

starting from any $n$.

Now $n, E$ and $n, F$ have identical operational behaviour. Since $0, E^{\prime}$ is unreachable, it is clear that

$\forall n . n, E \sim_{\mu} n, F$

holds. We also have $n+1, E^{\prime} \sim_{\mu} n+1, F^{\prime}$, for all $n$. However, $0, E^{\prime}$ and $0, F^{\prime}$ have distinct operational behaviour since we have $0, E^{\prime} \stackrel{z}{\rightarrow} 0, E$ as a transition.

Consider an atomic $\phi$ such that and $n, G \vDash \phi$ if and only if $n=0$ and $G$ is 1 , the unit process. Using the semantic clauses for $*$ and $\langle-\rangle$, we can verify that $1, E^{\prime} \vDash \phi *\langle z\rangle \top$ and $1, F^{\prime} \not \models \phi *\langle z\rangle \top$ since only $0,1 \vDash \phi$ and $1,1 \times E^{\prime} \stackrel{z}{\rightarrow} 1,1 \times E \vDash \top$ but $0,1 \times F^{\prime}$ makes no $z$-transition. We therefore have that

$$
0, E \vDash\langle i\rangle(\phi *\langle z\rangle \top) \quad 0, F \not \models\langle i\rangle(\phi *\langle z\rangle \top)
$$

hold.

This tells us that Theorem 9.1 of [PT06] fails as stated in the presence of both $\langle-\rangle$ and $*$. It also tells us that the operational equivalence $\sim_{\mu}$ is not contained in (or equal to) the logical equivalence relation.

\section{Corrections to Formal Aspects of Computing (2006) 18:495-517, [PT06]}

We must amend Definition 1, and make the necessary consequent amendments. The significance of the amendment is explained in Sect. 4.

Definition 1, p. 502, should be stated as follows:

Bisimulation, $\sim_{\mu}$, is the largest binary relation on processes $E$ such that if $E \sim_{\mu} F$, then

(i) for all $R, R, E \stackrel{a}{\longrightarrow} \mu(a, R), E^{\prime}$ implies, for some $F^{\prime}$,

$$
R, F \stackrel{a}{\longrightarrow} \mu(a, R), F^{\prime} \text { and } E^{\prime} \sim_{\mu} F^{\prime} \text {, and }
$$

(ii) for all $R, R, F \stackrel{a}{\longrightarrow} \mu(a, R), F^{\prime}$ implies, for some $E^{\prime}$,

$$
R, E \stackrel{a}{\longrightarrow} \mu(a, R), E^{\prime} \text { and } E^{\prime} \sim_{\mu} F^{\prime} .
$$

We refer to this bisimulation as global bisimulation.

For Theorem 9.2, however, we need the original definition of bisimulation. We use the symbol $\approx_{\mu}$ instead of $\sim_{\mu}$, and call it local bisimulation, to avoid confusion, as follows:

Local bisimulation, $\approx_{\mu}$, is the largest binary relation on resource-process pairs $R, E$ such that if $R, E \sim_{\mu} R, F$, then

(i) $\quad R, E \stackrel{a}{\longrightarrow} \mu(a, R), E^{\prime}$ implies, for some $F^{\prime}$,

$$
R, F \stackrel{a}{\longrightarrow} \mu(a, R), F^{\prime} \text { and } \mu(a, R), E^{\prime} \approx_{\mu} \mu(a, R), F^{\prime} \text {, and }
$$

(ii) $\quad R, F \stackrel{a}{\longrightarrow} \mu(a, R), F^{\prime}$ implies, for some $E^{\prime}$,

$$
R, E \stackrel{a}{\longrightarrow} \mu(a, R), E^{\prime} \text { and } \mu(a, R), E^{\prime} \approx_{\mu} \mu(a, R), F^{\prime} .
$$

The appropriate notion of congruence for this equivalence is then the following: if, for all $R, R, E \approx_{\mu} R, F$, then, for all evident terms, $a, X, G, R$, and $S$, we have $R, a: E \approx_{\mu} R, a: F, R, E+G \approx_{\mu} R, F+G, R, E \times G \approx_{\mu} R$, $F \times G$, and $R,(v S) E \approx_{\mu} R,(v S) F$.

This definition of local bisimulation should be inserted at the end of Sect. 4.

The following amendments then arise as consequences: 
- All assertions of the form $R, E \sim_{\mu} R, F$ should be replaced by the corresponding $E \sim_{\mu} F$ except those in Theorem 9.2;

- Theorem 9.1, p. 512, should be: "If $E \sim_{\mu} F$, then, for all $R$, it follows that $R, E \equiv_{\mathbf{M B I}} R, F$.";

- The first sentence of the paragraph immediately before Theorem 9.2 should be: "It follows that, for imagefinite processes, the argument of Stirling [Sti01] can be applied rather straightforwardly provided we use the local bisimulation, $\approx_{\mu}$, including for the definition of the logical connectives as given in Table 2.";

- Theorem 9.2, p. 513, should be: "If, for all $R, R, E$ and $R, F$ are image-finite and if, for all $R$, it is the case that $R, E \equiv_{\text {MBI }} R, F$, then $E \approx_{\mu} F$."

Known typographical errors:

- $\quad$ p. 509, 1. -19: " $R \sqsubseteq e$ " should be " $e \sqsubseteq R$ ";

- p. $513,1.6$ : " $R \sqsubseteq e$ " should be " $e \sqsubseteq R$ ";

- p. $514,1.12$ : " $R$ ' should be " $\mu(a, R)$ ";

- p. 514, 1. 13: " $H$ " should be " $H_{i}$ ";

- $\quad$ p. 516: In [BT00a], "3:281-305" should be "8(6-7):377-393, 2001";

- p. 516: In [BT00b], "3:281-305" should be "8(6-7):395-414, 2001”.

\section{Consequences for the paper Electronic Notes in Theoretical Computer Science 172, 545-587, 2007, [PT07]}

In all sections except Sect. 5, we need to make the same amendments as for [PT06]. Specifically:

Definition 3.1, p. 555, should be stated as follows:

Bisimulation, $\sim_{\mu}$, is the largest binary relation on processes $E$ such that if $E \sim_{\mu} F$, then

(i) for all $R, R, E \stackrel{a}{\longrightarrow} \mu(a, R), E^{\prime}$ implies, for some $F^{\prime}$,

$$
R, F \stackrel{a}{\longrightarrow} \mu(a, R), F^{\prime} \text { and } E^{\prime} \sim_{\mu} F^{\prime} \text {, and }
$$

(ii) for all $R, R, F \stackrel{a}{\longrightarrow} \mu(a, R), F^{\prime}$ implies, for some $E^{\prime}$,

$$
R, E \stackrel{a}{\longrightarrow} \mu(a, R), E^{\prime} \text { and } E^{\prime} \sim_{\mu} F^{\prime} .
$$

For Sect. 5 and for Theorems 8.3 and 9.2, however, we need the original bisimulation. We use the symbol $\approx_{\mu}$ instead of $\sim_{\mu}$, and call it local bisimulation, to avoid confusion, as follows:

Local bisimulation, $\approx_{\mu}$, is the largest binary relation on resource-process pairs $R, E$ such that if $R, E \sim_{\mu} R, F$, then

(i) $R, E \stackrel{a}{\longrightarrow} \mu(a, R), E^{\prime}$ implies, for some $F^{\prime}$,

$$
R, F \stackrel{a}{\longrightarrow} \mu(a, R), F^{\prime} \text { and } \mu(a, R), E^{\prime} \approx_{\mu} \mu(a, R), F^{\prime}, \text { and }
$$

(ii) $\quad R, F \stackrel{a}{\longrightarrow} \mu(a, R), F^{\prime}$ implies, for some $E^{\prime}$,

$$
R, E \stackrel{a}{\longrightarrow} \mu(a, R), E^{\prime} \text { and } \mu(a, R), E^{\prime} \approx_{\mu} \mu(a, R), F^{\prime} .
$$

The appropriate notion of congruence for this equivalence is then the following: if, for all $R, R, E \approx_{\mu} R, F$, then, for all evident terms, $a, X, G, R$, and $S$, we have $R, a: E \approx_{\mu} R, a: F, R, E+G \approx_{\mu} R, F+G, R, E \times G \approx_{\mu} R, F \times G$, $R,(v S) E \approx_{\mu} R,(v S) F$, and $R$, fix $X . E \approx_{\mu} R, \operatorname{fix} X . F$.

This definition of local bisimulation should be inserted at the end of Sect. 3.

The following amendments then arise as consequences:

- In Sect. 5: Each occurence of " $\sim_{\mu}$ " should be " $\approx_{\mu}$ ";

- All assertions of the form $R, E \sim_{\mu} R, F$ should be replaced by the corresponding $E \sim_{\mu} F$, except in Sect. 5 and in Theorem 8.3 and its immediately preceding paragraph;

- Theorem 8.2, p. 575, should be: "If $E \sim_{\mu} F$, then, for all $R$, it follows that $R, E \equiv_{\text {MBI }} R, F$."; 
- p. $577,1 .-4:$ " $\sim_{\mu}$ " should be “ $\approx_{\mu}$ ";

- p. 577, 1. -3: The sentence beginning "For now, ..." should be: "For now, however, we establish the basic result for image-finite processes, with the argument following that of Stirling [Sti01] rather straightforwardly provided we use the local bisimulation, $\approx_{\mu}$, including for the definition of the logical connectives as given in Table 5.";

- $\quad$ Theorem 8.3, p. 578, should be: "If, for all $R, R, E$ and $R, F$ are image-finite and if, for all $R$, it is the case that $R, E \equiv_{\text {MBI }} R, F$, then $E \approx_{\mu} F$."

The extensions of Theorems 8.2 and 8.3 given in Sect. 9, namely Theorems 9.1 and 9.2, require corresponding amendments.

- Theorem 9.1. p. 580, should be: "For all $R$, if $E \sim_{\mu} F$, then $R, E \equiv_{\text {MBI }} R, F$."

- Theorem 9.2, p. 580, should be: "Let $R, E$ and $R, F$ be image-finite. If, for all $R, R, E \equiv_{\mathrm{MBI}} R, F$, then $E \approx_{\mu} F$."

The significance of these amendments is explained in Sect. 4.

Finally, the following typographical corrections are known:

- p. 572, 1. -21: " $R \sqsubseteq e$ " should be " $e \sqsubseteq R$ ";

- p. 576, 1. -15: " $R \sqsubseteq e$ " should be " $e \sqsubseteq R$ ";

- p. 578,1 . -13: " $R$ ” should be “ $\mu(a, R)$ ”.

\section{Discussion}

Our (non-typographical) corrections are all based on the necessary correction to the definition of bisimulation required for the logical equivalence theorems to hold (for the full MBI logic). Notice, however, that our original definition of bisimulation is the correct one for our denotational semantics, given in Sect. 5 of [PT07], which corresponds to the operational behaviour of SCRP.

So, there is a mismatch between between the operational behaviour of SCRP and the logical strength of full MBI. In particular, as we have seen in Sect. 1, a mismatch occurs when $*$ and $\langle-\rangle$ are both present in the logic. There exist fragments of MBI, however, for which the logical equivalence theorems hold for both $\sim_{\mu}$ and $\approx_{\mu}$ : for example, MBI without $*$ and the multiplicative quantifiers and modalities but, importantly, with $*$ and the additive modalities. Indeed, such fragments appear to be useful in practical modelling work.

Notice that we have renamed the original notion of bisimulation, now denoted $\approx_{\mu}$, as "local". This is in contrast to the "global" form required for (one direction of) the logical equivalence result. For instance, in the example of Sect. 1, the local equivalence is available but the global one is not, so that logical equivalence fails. The local equivalence holds because the states $0, E^{\prime}$ and $0, F^{\prime}$ are not reachable in the transition system and the global one fails because the quantification over all resources includes 0 .

The mismatch shows that the expressivity of MBI exceeds that which is captured by the local equivalence. In particular, again referring to the example of Sect. 1, the local equivalence fails to distinguish the perturbations, $0, E^{\prime}$ and $0, F^{\prime}$, of $1, E^{\prime}$ and $1, F^{\prime}$ (which are not logically equivalent). The global equivalence distinguishes such perturbations.

Finding a bisimulation that yields both the logical equivalence theorem for full MBI and an internal full abstraction theorem, so that we might obtain an appropriate account of "domain theory in logical form" in our setting, appears to be a challenging problem.

\section{References}

[PT06] Pym D, Tofts C (2006) A calculus and logic of resources and processes. Formal Aspects Computing 18(4):495-517

[PT07] Pym D, Tofts C (2007) Systems modelling via resources and processes: philosophy, calculus, semantics, and logic. In: Cardelli L, Fiore M, Winskel G (eds) Electronic notes in theoretical computer science (Computation, Meaning, and Logic: Articles dedicated to Gordon Plotkin), vol 107, p 545-587

Communicated 26 July 2007 by J. V. Tucker

Published online 19 September 2007 